\title{
Fast Molecular Compression by a Hyperthermal Collision Gives Bond-Selective Mechanochemistry
}

\author{
Lukas Krumbein, ${ }^{1, \dagger}$ Kelvin Anggara $\odot,{ }^{1, \dagger}$ Martina Stella $\odot,{ }^{2}$ Tomasz Michnowicz, ${ }^{1}$ Hannah Ochner $\odot,{ }^{1}$ \\ Sabine Abb, ${ }^{1}$ Gordon Rinke, ${ }^{1}$ André Portz, ${ }^{3}$ Michael Dürr, ${ }^{3}$ Uta Schlickum, ${ }^{1,4}$ Andrew Baldwin, ${ }^{5}$ \\ Andrea Floris, ${ }^{6}$ Klaus Kern, ${ }^{1,7}$ and Stephan Rauschenbach ${ }^{1,5, *}$ \\ ${ }^{1}$ Max-Planck-Institut für Festkörperforschung, Heisenbergstrasse 1, DE-70569 Stuttgart, Germany \\ ${ }^{2}$ Department of Materials, Royal School of Mines, Imperial College London, Exhibition Road, London SW7 2A2, United Kingdom \\ ${ }^{3}$ Institut für Angewandte Physik, Justus-Liebig-Universität Giessen, Heinrich-Buff-Ring 16, DE-35392 Giessen, Germany \\ ${ }^{4}$ Institut für Angewandte Physik, Technische Universität Braunschweig, Mendelssohnstrasse 2, DE-38106 Braunschweig, Germany \\ ${ }^{5}$ Department of Chemistry, Chemistry Research Laboratory, University of Oxford, \\ 12 Mansfield Road, Oxford OX1 3TA, United Kingdom \\ ${ }^{6}$ School of Chemistry, University of Lincoln, Brayford Pool, Lincoln LN6 7TS, United Kingdom \\ ${ }^{7}$ Institut de Physique, École Polytechnique Fédérale de Lausanne, Laussane CH-1015, Switzerland
}

(Received 14 July 2020; accepted 7 December 2020; published 1 February 2021)

\begin{abstract}
Using electrospray ion beam deposition, we collide the complex molecule Reichardt's dye $\left(\mathrm{C}_{41} \mathrm{H}_{30} \mathrm{NO}^{+}\right)$ at low, hyperthermal translational energy $(2-50 \mathrm{eV})$ with a $\mathrm{Cu}(100)$ surface and image the outcome at single-molecule level by scanning tunneling microscopy. We observe bond-selective reaction induced by the translational kinetic energy. The collision impulse compresses the molecule and bends specific bonds, prompting them to react selectively. This dynamics drives the system to seek thermally inaccessible reactive pathways, since the compression timescale (subpicosecond) is much shorter than the thermalization timescale (nanosecond), thereby yielding reaction products that are unobtainable thermally.
\end{abstract}

DOI: 10.1103/PhysRevLett.126.056001

Chemistry is concerned with the manipulation of bonds between atoms with the goal to use chemical reactions to form desired substances. Achieving this goal requires an understanding of how selectivity emerges. Chemical reactions that occur following molecule-surface collisions are, technologically and fundamentally, important in fields as diverse as heterogeneous catalysis [1-9], epitaxial material fabrication [10-12], biomolecular analysis [13-17], and astrochemistry [10]. Bond-selective reactions in a molecule-surface collision have been demonstrated by exciting specific vibrational modes of a molecule right before its surface impact $[5,7,8]$. This approach succeeds due to the sudden energy accumulation in a specific molecular degree of freedom triggering a reaction that promptly occurs before the deposited energy spreads to other degrees of freedom not involved in the reaction, i.e., before thermalization $[18,19]$.

Imparting energy into the molecular center-of-mass motion (i.e., translation) toward the surface offers an alternative means to induce surface reactions, which is

Published by the American Physical Society under the terms of the Creative Commons Attribution 4.0 International license. Further distribution of this work must maintain attribution to the author(s) and the published article's title, journal citation, and DOI. Open access publication funded by the Max Planck Society. attractive because translational energy of a molecular ion is readily achieved by acceleration in an electric field. However, simply accelerating molecules toward the surface has been deemed unsuitable for obtaining bond-selective reactions, because the collision excites soft modes of the molecule $[20,21]$, which are poorly coupled to stiff stretching modes that promote bond-breaking reactions $[9,19]$. As a result, reactions would happen late after the thermalization occurs, which diverges reaction pathways toward nonselective outcomes [10,22-26].

It remains unclear whether excited soft modes in the absence of thermalization could give a selective reaction because previous studies have only detected the reaction products in the gas phase [10,22-26], whereby excess energy from the collision cannot be removed from the molecular product and subsequently causes further reactions. We avoid this issue by using the surface to remove excess energy from the collision products. This is readily achieved by carrying out the experiment at lower, nearthreshold energies, which necessitates the detection of the adsorbed collision outcome on surface.

Here we show that the large excitation of soft modes in a molecule-surface collision, i.e., extensive compression of a molecule, occurring at timescales faster than thermalization, leads to a selective, nonthermal reaction path. We have used scanning tunneling microscopy (STM) to detect the surfacebound products from collisions between a large molecular 
ion and a metal surface, carried out at low hyperthermal energies $(2-50 \mathrm{eV})$. In our experiment, singly protonated Reichardt's dye ( $\mathrm{RD}, \mathrm{C}_{41} \mathrm{H}_{30} \mathrm{NO}^{+}$) was collided at normal incident angle with a $\mathrm{Cu}(100)$ surface held at room temperature using electrospray ion beam deposition [14,27,28]. The collision outcome, examined by STM at $11 \mathrm{~K}$ (see Supplemental Material Fig. S1 [28]), revealed reactive pathways that selectively cleaved a single $\mathrm{C}-\mathrm{N}$ bond in RD. Energy-dependent experiments and ab initio molecular dynamics (MD) calculations revealed orientation-dependent dynamics that selectively bend specific $\mathrm{C}-\mathrm{N}$ bonds in the molecule, ignoring the minimum energy path.

Our Letter gives insight into the emergence of bondselective mechanochemical reactions in molecule-surface collisions. Collision-induced mechanochemistry [50,51] promises to be generally applicable to large molecules such as peptides or even proteins [13-17], providing a new tool to perform nonthermal on-surface synthesis of novel molecular materials.

Experiment.-Figure 1 shows three outcomes obtained from the collision between a RD ion and a $\mathrm{Cu}(100)$ surface: one nonreactive and two reactive outcomes, as revealed by STM imaging and simulation. These are the outcomes of specific collision dynamics that are accessed by aiming the ion beam at normal incidence angle to the surface, as shown in Fig. 1(a). These three pathways were obtained by analyzing the majority $(\sim 80 \%)$ of species on the surface. The remaining $\sim 20 \%$ of the adsorbate were excluded from our analysis because they cannot be clearly classified (see Supplemental Material [28]). These excluded features can be due to atypical conformations of the identified species at defects, step edges, or in clusters, or they may indicate additional pathways beyond the three discussed here.

The nonreactive pathway, "intact," was found to yield an adsorbed intact RD with its two phenyl rings oriented vertical from the surface, as shown in Fig. 1(b). We expect the proton that was attached to the $\mathrm{O}$ atom in the $\mathrm{RD}$ ion to undergo spontaneous dehydrogenation on surface at room temperature given the low computed barrier of $0.08 \mathrm{eV}$.

The reactive pathways were found to be bond selective to the $\mathrm{C}-\mathrm{N}$ bonds, breaking either one out of the two types of $\mathrm{C}-\mathrm{N}$ bonds in $\mathrm{RD}$ [see Fig. 1(a)]: the $\mathrm{C}-\mathrm{N}$ bond pointing at an angle "across" the $\mathrm{N}-\mathrm{O}$ axis of the molecule, termed $\mathrm{C}-\mathrm{N}(\mathrm{AC})$, or the $\mathrm{C}-\mathrm{N}$ bond pointing "along" the $\mathrm{N}-\mathrm{O}$ axis, termed $\mathrm{C}-\mathrm{N}(\mathrm{AL})$. In the "crack" pathway, a single $\mathrm{C}-\mathrm{N}(\mathrm{AC})$ was broken to give a dissociated RD fragment ( $\alpha$ fragment), as shown in Fig. 1(b). In the "split" pathway, the $\mathrm{C}-\mathrm{N}(\mathrm{AL})$ bond was broken to give two products, as shown in Fig. 1(b): a diphenylphenoxy ( $\beta$ fragment) and a triphenylpyridine ( $\Delta$ fragment). The observation of crack and split pathways thus establishes the existence of bondselective pathways due to molecule-surface collision at hyperthermal translational energy.

To gain insight into the dynamics of these bondselective pathways, we measured their respective reaction
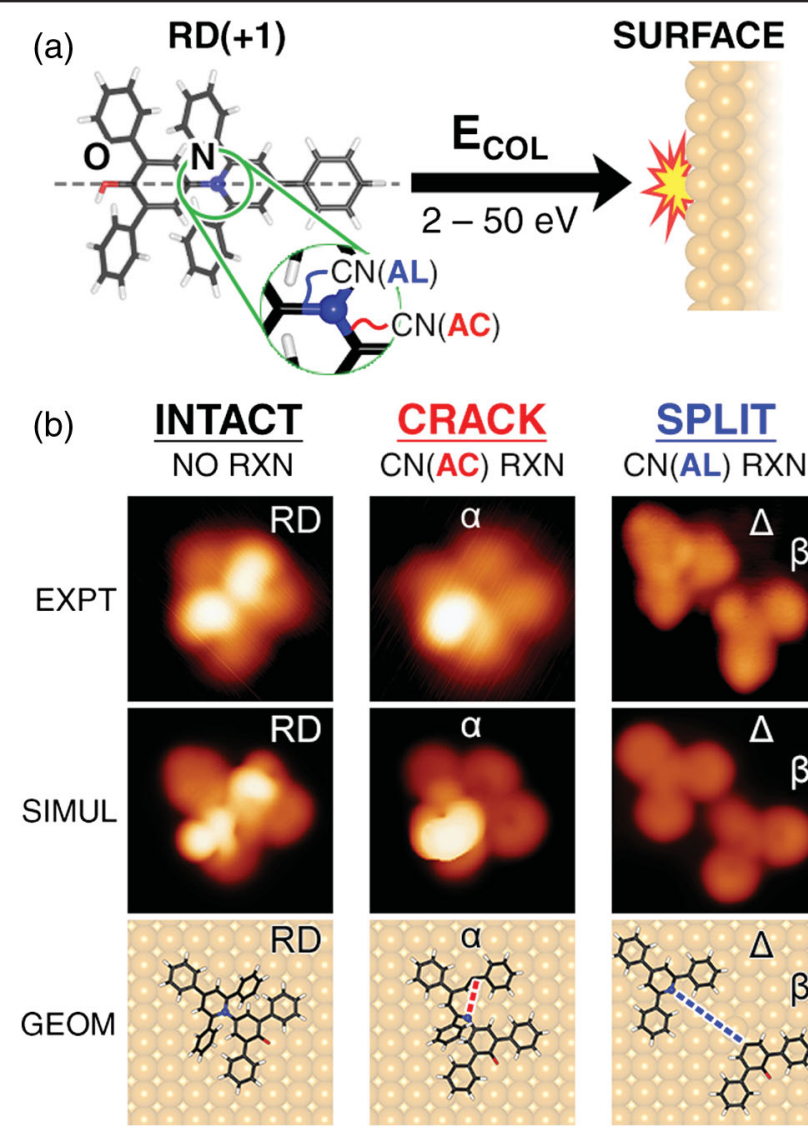

CRACK


FIG. 1. Hyperthermal collision of RD on $\mathrm{Cu}(100)$ surface. (a) Schematics of the experiment, showing a beam of singly protonated $\mathrm{RD}(+1)$ aimed along the surface normal to the $\mathrm{Cu}$ surface at room temperature. Two types of $\mathrm{C}-\mathrm{N}$ bond are labeled as $\mathrm{C}-\mathrm{N}(\mathrm{AC})$ and $\mathrm{C}-\mathrm{N}(\mathrm{AL})$ based on their orientation against the N-O axis in RD (gray dashed line). (b) STM image (EXPT) and simulation (SIMUL) of the three collision outcomes imaged at $11 \mathrm{~K}$. The intact pathway gives adsorbed RD. The crack pathway breaks a $\mathrm{CN}(\mathrm{AC})$ bond in the parent $\mathrm{RD}$ to give one $\alpha$ fragment, while the split pathway breaks the $\mathrm{CN}(\mathrm{AL})$ bond to give one $\beta$ and one $\Delta$ fragment. Computed geometries (GEOM) show broken $\mathrm{CN}(\mathrm{AC})$ bond (red dashed line) in the $\alpha$ fragment and broken $\mathrm{CN}(\mathrm{AL})$ bond (blue dashed line) between $\beta$ and $\Delta$ fragments.

probabilities against the kinetic energy of the molecular beam. We varied the collision energy by decelerating the ions approaching the surface, which shifted the energy distribution of the ion beam without changing its width (see Supplemental Material Methods and Fig. S3 [28]). Since the ion beam is aimed normal to the surface, the collision energy is the kinetic energy, which corresponds to the molecular translation along the surface normal. The probability of each pathway was obtained by counting the number of species present on surface (see Supplemental Material for details of the analysis, Fig. S2). The result of this energy-dependent measurement was fitted to a model, inspired by the sudden vector projection description of molecular collisions at the gas-surface interface [19]. 


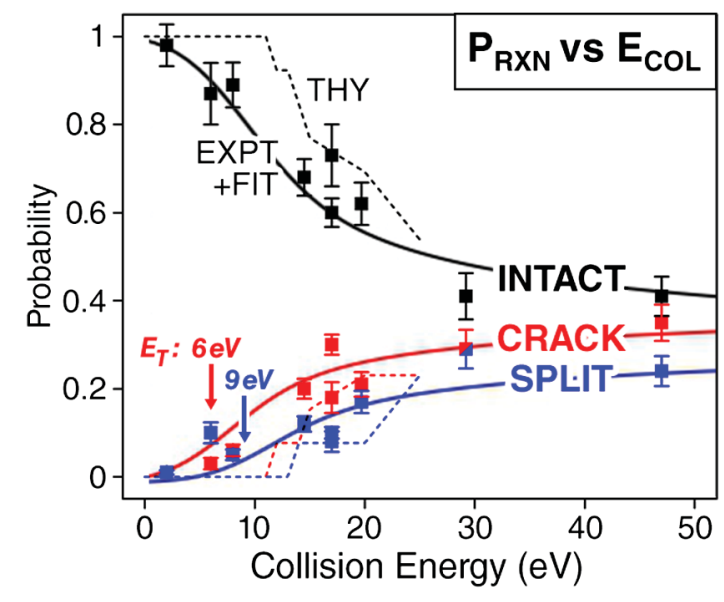

FIG. 2. Evidence of reactive collision by incident translational energy. Probabilities to observe intact (black), crack (red), and split (blue) outcomes measured against the collision energy. The data points (square) were fitted with a reaction probability model [solid line, Eq. (1)] to give threshold energy $\left(E_{T}\right)$ for each pathway (see Methods for details). Dashed line shows probability obtained from $a b$ initio MD calculations. Error bars show standard deviation.

We compute the reaction probability $\left(P_{\mathrm{RXN}, X}\right)$ for each pathway $(X)$ by projecting the molecular translation vector to the reaction coordinate vector to estimate how much of the translation energy $\left(E_{\mathrm{COL}}\right)$ is utilized to propel the system toward the transition state (see Supplemental Material Fig. S4 and Methods for details). The model yields the relation

$$
P_{X}\left(E_{\mathrm{COL}}\right)=P_{\mathrm{SAT}, X} \cos ^{-1} \sqrt{\frac{E_{T, X}}{E_{\mathrm{COL}}}} .
$$

The fitting gave a translational energy threshold $\left[E_{T}\right.$ in Eq. (1)] of $6.0 \mathrm{eV}$ for crack and $9.5 \mathrm{eV}$ for split, as shown in Fig. 2. The existence of this threshold, which marks the minimal energy needed for the reaction, thereby evidences the translational energy as the cause of the reaction, ruling out surface-to-molecule charge transfer $[10,24,25]$ as a sole cause of the reaction.

Further dynamical insight is obtained by the observation of the reaction probabilities approaching a limiting value $P_{\mathrm{SAT}, X}$, estimated to be 0.43 for crack and 0.35 for split at high energies using Eq. (1). Such a saturation of reaction probability below unity has been observed in gas-surface scattering experiment $[25,52,53]$ and in surface-induced dissociation of proteins [16]. From the diatomic-surface scattering studies $[52,53]$, the saturation was understood to be due to a steric effect in which there was a limited range of orientations in the approaching molecule (also known as "cone of acceptance"; see Ref. [54]) that upon collision with the surface would lead to a reactive outcome. Such a strong dependence on initial orientation is characteristic of a direct reaction in which a single-collision event causes a direct energy transfer from the molecule translation into the reaction coordinate $[1,52,53,55]$. Transferring insights from the diatomic-surface scattering studies to the present Letter, the saturation in reaction probability observed for crack and split is therefore indicative of an orientation-dependent direct reaction caused by a single-collision event, ruling out a multicollision event $[55,56]$ via a precursor state [57].

Theory.-We corroborate this experimental inference by simulating the $\mathrm{RD}-\mathrm{Cu}(100)$ collision by MD calculations, which revealed that crack and split are orientation-dependent direct reactions caused by a single-collision event [see Figs. 3(a) and 4, Supplemental Material [28], Videos 1 and 2]. The collision was modeled as a positively charged RD ion approaching a negatively charged surface that was understood to contain the image charge of the RD ion (see Supplemental Material Fig. S5). By varying (i) the initial translational energy along the surface normal and (ii) the initial orientation of the incident molecule [see Fig. 3(a), shown as the surface approaching the molecule], the calculations reproduced the three outcomes observed in the experiment: the nonreactive intact pathway and the bond-selective crack and split pathways, with a threshold of $12 \mathrm{eV}$ for crack and $14 \mathrm{eV}$ for split. In our limited sampling of 26 collision geometries [see Fig. 3(a)], the calculations did not reveal additional reaction pathways beyond intact, crack, and split. Most notably, the computed reaction probabilities were found to reproduce the experimental trend, as shown in Fig. 2 and Supplemental Material Fig. S6, thereby validating the collision dynamics unveiled by the MD.

The collision dynamics for all three pathways, in general, showed the molecule to be compressed onto the surface due to the mechanical impulse from the molecule-surface impact. As shown in Fig. 3(b), the stopping force exerted by the surface on the incident molecule was computed to be operative in the nano-Newton regime at subpicosecond timescale (see also Supplemental Material Fig. S7 [28]). This impulse is understood to move the system to a region on the potential energy surface along the compression coordinate. For the reactive crack and split outcome, the system was impulsively propelled to a transition state en route to a product potential well.

The computed dynamics for crack and split show the collision-induced compression precedes the bond-selective dissociation (see Fig. 4 and Supplemental Material Videos 1 and 2 [28]). The compression bends a specific $\mathrm{C}-\mathrm{N}$ bond [i.e., $\mathrm{C}-\mathrm{N}(\mathrm{AC})$ in crack and $\mathrm{C}-\mathrm{N}(\mathrm{AL})$ in split] prior to its dissociation (see bottom panels of Fig. 4). This $\mathrm{C}-\mathrm{N}$ bond bending was noted to modify the local geometry of the $\mathrm{C}$ atom from a trigonal planar ( $s p^{2} \mathrm{C}$ atom) to a trigonal pyramidal ( $s p^{3} \mathrm{C}$ atom), in effect converting a $p$ orbital into a $s p^{3}$-hybridized $\mathrm{C}$-dangling bond. Upon contact with the surface, this bent $\mathrm{C}-\mathrm{N}$ bond breaks, and concurrently a new $\mathrm{C}-\mathrm{Cu}$ bond forms, indicating a reactive event whose transition state is stabilized by the formation of a new bond 

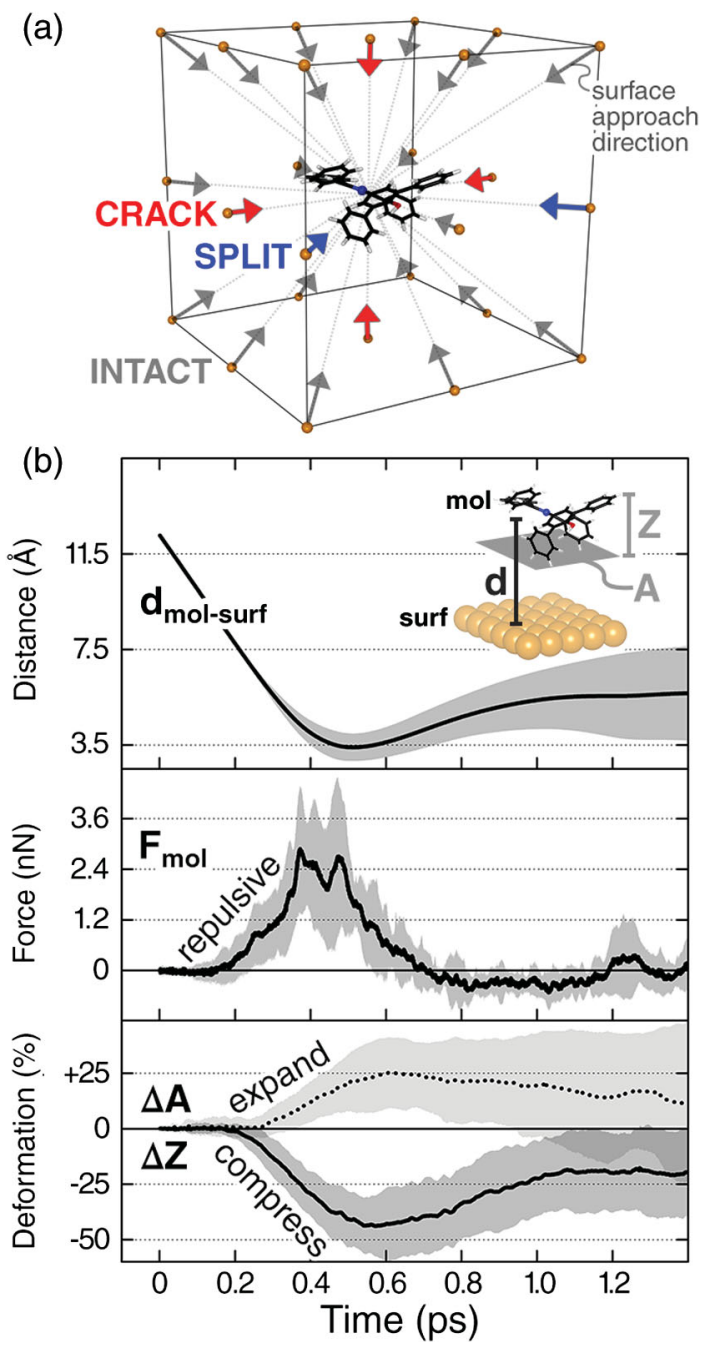

FIG. 3. Computed dynamics of RD-Cu(100) collision with varied initial geometries. (a) Schematics of 26 different initial RD geometries simulated for $15 \mathrm{eV}$ collision energy, illustrated here as the surface approaching the molecule (arrows mark the surface normal). Red and blue arrows indicate initial geometries that give crack and split outcomes, respectively; gray arrows for intact. (b) Time-dependent quantities of RD averaged between all trajectories at $15 \mathrm{eV}$ incident energy with different initial geometries: molecule-surface distance $\left(\boldsymbol{d}_{\text {mol-surf }}\right)$, stopping force exerted by the surface on RD $\left(\boldsymbol{F}_{\text {mol }}\right)$, change in RD footprint along the surface plane direction $(\boldsymbol{\Delta} \boldsymbol{A}$, dotted line), and the compression of RD along the surface normal $(\Delta Z$, solid line). Shaded area is standard deviation of all trajectories.

[58]. In both pathways, the trajectory shows the $\mathrm{C}-\mathrm{N}$ bond to dissociate in a single attempt, evidencing a direct energy transfer from the translational energy to the reaction coordinate.

The preferential breaking of the $\mathrm{C}-\mathrm{N}$ bond for the bondselective crack and split pathways was understood to be due to its pronounced reactivity, which we attribute to two factors: (i) the destabilization due to $\mathrm{C}-\mathrm{N}$ bending and (ii) the $\mathrm{C}-\mathrm{N}$ antibonding orbital being the lowest unoccupied molecular orbital (LUMO) of RD (see Supplemental

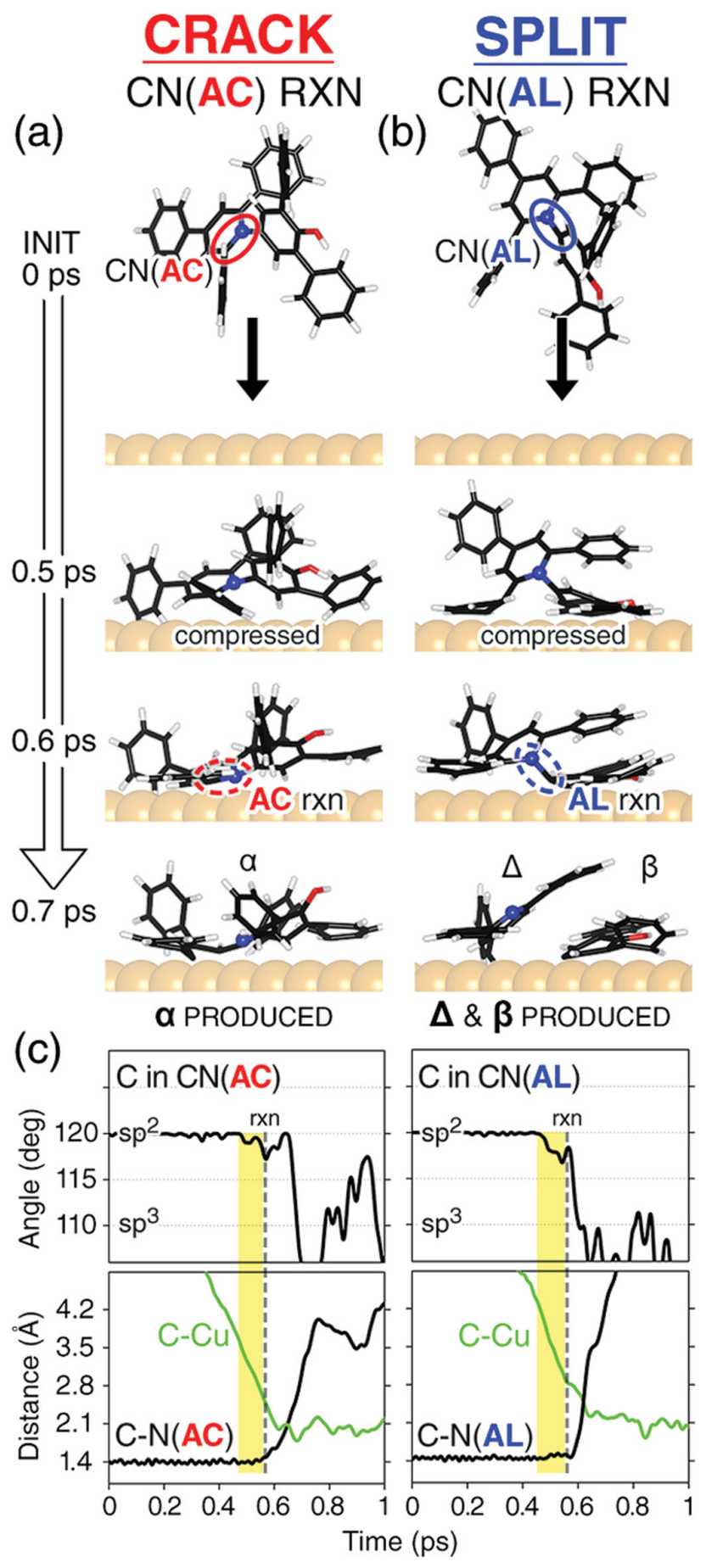

FIG. 4. Reactive trajectories for crack and split in RD-Cu(100) collision. Time-dependent snapshots of the (a) crack and (b) split pathway. Trajectories shown are for the minimum translational energy required to give reactive outcome: $12 \mathrm{eV}$ for crack pathway and $14 \mathrm{eV}$ for split. (c) Time-dependent quantities of RD collision: average angle formed between the $\mathrm{C}$ atom in $\mathrm{C}-\mathrm{N}$ and two of its neighboring atoms, which checks whether the $\mathrm{C}$ atom in the $\mathrm{C}-\mathrm{N}$ bond is $s p^{2}$ - or $s p^{3}$-like; $\mathrm{C}-\mathrm{N}$ distance gives the separation between atoms in the reacting $\mathrm{C}-\mathrm{N}$ bond (black line); separation between the $\mathrm{C}$ atom of reacting $\mathrm{C}-\mathrm{N}$ bond and nearest $\mathrm{Cu}$ atom (green line). The yellow area marks the region where compression from the collision bends the $\mathrm{C}-\mathrm{N}$ bonds; the dashed line marks the beginning of the $\mathrm{C}-\mathrm{N}$ rupture. 
Material Fig. S8 [28]). The importance of these two factors were suggested by the correlation between $\mathrm{C}-\mathrm{N}$ dissociation and the charge flow from the surface to the LUMO of the compressed RD (i.e., RD with bent $\mathrm{C}-\mathrm{N}$ bonds) (see Supplemental Material Fig. S9). Similar to the mechanism proposed for $\mathrm{C}-\mathrm{H}$ dissociation of benzene on $\mathrm{Cu}$ surface [59], we expect the orbital mixing caused by the $\mathrm{C}-\mathrm{N}$ bond bending increases the propensity of the RD LUMO to hybridize and forms a bond with the surface. The proposed mechanism in which bond bending alters the electronic structure of a reacting molecule is noted to be topical in mechanochemistry [50,51].

Finally, to address the possibility of the hyperthermal collision giving a chemical reaction via a precursor state of $\mathrm{RD}$, we examined the minimum energy pathway (MEP) of an adsorbed RD. We consider this alternative pathway because an incident RD that had failed to react upon its first collision would have its energy increasingly equipartitioned among all its degrees of freedom while being trapped on surface, allowing the system to search for the MEP to react. The computed barrier along the MEP from an adsorbed RD to give an $\alpha$ fragment was $1.23 \mathrm{eV}$ and to give $\beta$ and $\Delta$ fragments was $0.28 \mathrm{eV}$ (see Supplemental Material Fig. S10 [28]). The MEP barrier thus predicts a reactive outcome dominated by the $\beta$ and $\Delta$ fragments. To test this prediction, we annealed a surface containing adsorbed $\mathrm{RD}$ at $\sim 350 \mathrm{~K}$ to initiate its thermal reaction (see Supplemental Material Fig. S11). The reaction was found to generate only $\beta$ and $\Delta$ fragments as products, in agreement with the MEP prediction. The hyperthermal collision clearly deviates from the MEP prediction since the $\alpha$ fragment was the major product, thereby ruling out reactions via precursor state.

In conclusion, we have reported here the first observation of a bond-selective reaction from a hyperthermal collision of a polyatomic ion with a metal surface. The collision gave a mechanical impulse that compresses the incident molecule, causing a specific chemical bond to be activated. From a fundamental standpoint, we expect the collisioninduced molecular compression described here to be a general phenomenon across surfaces with similar stiffness; we expect such mechanism to play a central role in surfaceinduced dissociation experiments in tandem mass spectroscopy [13-17]. The experimental method described here provides a new general tool to study and apply compressive mechanochemistry for any molecules that can be electrosprayed [27]. Here the compression is operative on the entire molecule, instead of locally [60], for subpicoseconds, instead of permanently [51], thereby opening a new avenue to explore impulsive mechanochemistry. Our current instrumentation is limited by the finite spread of the translational energy in the incident molecule, which introduces uncertainty in the measured threshold energies and in the details of how reaction channels are opened. Furthermore, the control of molecular orientation prior to its surface collision either by using optical [61] or hexapole field [62] should enable selection of reaction pathway. The method described here thus offers a new scalable method to nonthermally activate molecules to generate species that are inaccessible from conventional thermochemistry $[12,27]$.

The authors declare that the data supporting the findings of this study are available from the corresponding author upon request.

Special thanks to Alessandro De Vita, who should have been among the authors. We thank Rico Gutzler, John Polanyi, Claire Vallance, Mark Brouard, Christian Reichardt for fruitful discussions. Calculations were performed on the supercomputers at Max-Planck Computing and Data Facility in Garching. The research is funded by the Max-Planck Society. K. A. thanks Alexander von Humboldt Foundation for financial support. Via our membership of the UK's HEC Materials Chemistry Consortium, which is funded by EPSRC (EP/L000202, EP/R029431), this work used the ARCHER UK National Supercomputing Service [63] and the UK Materials and Molecular Modelling Hub for computational resources, MMM Hub, which is partially funded by EPSRC (EP/P020194). S. R. designed and supervised the project. S. R., S. A., K. K., and U.S. planned experiments. L. K., S. R., T. M., G. R., and K. A. performed the experiments. L. K., S. R. H. O., K. A., S. A., and A. B. analyzed experimental data. U.S., T. M., A. P., and M. D. performed chemical analysis measurement. K. A., M.S., and A.F. performed the DFT calculations. The authors declare no competing financial interests.

*Corresponding Author. stephan.rauschenbach@chem.ox.ac.uk ${ }^{\dagger}$ L. K. and K. A. contributed equally to this work.

[1] S. T. Ceyer, Science 249, 133 (1990).

[2] H. Hou, S. J. Gulding, C. T. Rettner, A. M. Wodtke, and D. J. Auerbach, Science 277, 80 (1997).

[3] R. D. Beck, P. Maroni, D. C. Papageorgopoulos, T. T. Dang, M. P. Schmid, and T. R. Rizzo, Science 302, 98 (2003).

[4] R. R. Smith, D. R. Killelea, D. F. DelSesto, and A. L. Utz, Science 304, 992 (2004).

[5] D. R. Killelea, V. L. Campbell, N. S. Shuman, and A. L. Utz, Science 319, 790 (2008).

[6] B. L. Yoder, R. Bisson, and R. D. Beck, Science 329, 553 (2010).

[7] L. Chen, H. Ueta, R. Bisson, and R. D. Beck, Faraday Discuss. 157, 285 (2012).

[8] X. J. Shen, A. Lozano, W. Dong, H. F. Busnengo, and X. H. Yan, Phys. Rev. Lett. 112, 046101 (2014).

[9] B. Jiang, M. Yang, D. Xie, and H. Guo, Chem. Soc. Rev. 45, 3621 (2016).

[10] D. C. Jacobs, Annu. Rev. Phys. Chem. 53, 379 (2002).

[11] J. V. Barth, G. Costantini, and K. Kern, Nature (London) 437, 671 (2005). 
[12] G. Dubey, R. Urcuyo, S. Abb, G. Rinke, M. Burghard, S. Rauschenbach, and K. Kern, J. Am. Chem. Soc. 136, 13482 (2014).

[13] A. R. Dongré, Á. Somogyi, and V. H. Wysocki, J. Mass Spectrom. 31, 339 (1996).

[14] V. Grill, J. Shen, C. Evans, and R. G. Cooks, Rev. Sci. Instrum. 72, 3149 (2001).

[15] J. Laskin, T. H. Bailey, and J. H. Futrell, J. Am. Chem. Soc. 125, 1625 (2003).

[16] X. Ma, M. Zhou, and V. H. Wysocki, J. Am. Soc. Mass Spectrom. 25, 368 (2014).

[17] S. R. Harvey, J. T. Seffernick, R. S. Quintyn, Y. Song, Y. Ju, J. Yan, A. N. Sahasrabuddhe, A. Norris, M. Zhou, E. J. Behrman, S. Lindert, and V. H. Wysocki, Proc. Natl. Acad. Sci. U.S.A. 116, 8143 (2019).

[18] F. F. Crim, Proc. Natl. Acad. Sci. U.S.A. 105, 12654 (2008).

[19] H. Guo and B. Jiang, Acc. Chem. Res. 47, 3679 (2014).

[20] B. D. Kay, T. D. Raymond, and M. E. Coltrin, Phys. Rev. Lett. 59, 2792 (1987).

[21] K. Golibrzuch, J. H. Baraban, P. R. Shirhatti, J. Werdecker, C. Bartels, and A. M. Wodtke, Z. Phys. Chem. 229, 1929 (2015).

[22] J. A. Burroughs, S. B. Wainhaus, and L. Hanley, J. Phys. Chem. 98, 10913 (1994).

[23] R. D. Beck, J. Rockenberger, P. Weis, and M. M. Kappes, J. Chem. Phys. 104, 3638 (1996).

[24] W. R. Koppers, J. H. M. Beijersbergen, K. Tsumori, T. L. Weeding, P. G. Kistemaker, and A. W. Kleyn, Phys. Rev. B 53, 11207 (1996).

[25] J. R. Morris, G. Kim, T. L. O. Barstis, R. Mitra, and D. C. Jacobs, J. Chem. Phys. 107, 6448 (1997).

[26] Y. Yao, P. Shushkov, T. F. Miller, and K. P. Giapis, Nat. Commun. 10, 2294 (2019).

[27] S. Rauschenbach, M. Ternes, L. Harnau, and K. Kern, Annu. Rev. Anal. Chem. 9, 473 (2016).

[28] See Supplemental Material at http://link.aps.org/ supplemental/10.1103/PhysRevLett.126.056001 for full experimental and calculation details, which includes Refs. [29-49].

[29] V. G. Machado, R. I. Stock, and C. Reichardt, Chem. Rev. 114, 10429 (2014).

[30] J. Tersoff and D. R. Hamann, Phys. Rev. Lett. 50, 1998 (1983).

[31] J. Tersoff and D. R. Hamann, Phys. Rev. B 31, 805 (1985).

[32] L. N. Kantorovich (1996) User-friendly visualisation program for $a b$ initio DFT codes VASP, SIESTA, QE, and QUICKSTEP, https://nms.kcl.ac.uk/lev.kantorovitch/codes/lev00/index.html.

[33] G. Kresse and J. Hafner, Phys. Rev. B 47, 558 (1993).

[34] G. Kresse and J. Furthmüller, Phys. Rev. B 54, 11169 (1996).

[35] P. E. Blöchl, Phys. Rev. B 50, 17953 (1994).

[36] S. Grimme, J. Antony, S. Ehrlich, and H. Krieg, J. Chem. Phys. 132, 154104 (2010).

[37] K. Momma and F. Izumi, J. Appl. Crystallogr. 44, 1272 (2011).

[38] F. Neese, Comput. Mol. Sci. 2, 73 (2012).
[39] F. Weigend and R. Ahlrichs, Phys. Chem. Chem. Phys. 7, 3297 (2005).

[40] J. Zheng, X. Xu, and D. G. Truhlar, Theor. Chem. Acc. 128, 295 (2011).

[41] I. L. Geada, H. Ramezani-Dakhel, T. Jamil, M. Sulpizi, and H. Heinz, Nat. Commun. 9, 716 (2018).

[42] G. L. Stoychev, A. A. Auer, and F. Neese, J. Chem. Theory Comput. 13, 554 (2017).

[43] H. Jónsson, G. Mills, and K. W. Jacobsen, Nudged elastic band method for finding minimum energy paths transitions, in Classical and Quantum Dynamics in Condensed Phase Simulations, edited by B. J. Berne, G. Ciccotti, and D. F. Coker (World Scientific, Singapore, 1998), p. 385.

[44] G. Henkelman, B. P. Uberuaga, and H. Jónsson, J. Chem. Phys. 113, 9901 (2000).

[45] P. Giannozzi et al., J. Phys. Condens. Matter 21, 395502 (2009).

[46] G. Kresse and D. Joubert, Phys. Rev. B 59, 1758 (1999).

[47] J. P. Perdew, K. Burke, and M. Ernzerhof, Phys. Rev. Lett. 77, 3865 (1996).

[48] R. Sabatini, T. Gorni, and S. de Gironcoli, Phys. Rev. B 87, 041108(R) (2013).

[49] M. Methfessel and A. T. Paxton, Phys. Rev. B 40, 3616 (1989).

[50] J. J. Gilman, Science 274, 65 (1996).

[51] H. Yan, F. Yang, D. Pan, Y. Lin, J. N. Hohman, D. Solis-Ibarra, F. H. Li, J.E.P. Dahl, R. M. K. Carlson, B. A. Tkachenko, A. A. Fokin, P. R. Schreiner, G. Galli, W. L. Mao, Z.-X. Shen, and N. A. Melosh, Nature (London) 554, 505 (2018).

[52] E. Kolodney, A. Amirav, R. Elber, and R. B. Gerber, Chem. Phys. Lett. 111, 366 (1984).

[53] H. A. Michelsen, C. T. Rettner, D. J. Auerbach, and R. N. Zare, J. Chem. Phys. 98, 8294 (1993).

[54] R. D. Levine, Molecular Reaction Dynamics (Cambridge University Press, Cambridge, 2005).

[55] J. C. Polanyi and R. J. Wolf, Ber. Bunsenges. Phys. Chem. 86, 356 (1982).

[56] J. C. Polanyi and R. J. Wolf, J. Chem. Phys. 82, 1555 (1985).

[57] K. D. Rendulic and A. Winkler, Surf. Sci. 299-300, 261 (1994).

[58] L. Leung, T. Lim, Z. Ning, and J. C. Polanyi, J. Am. Chem. Soc. 134, 9320 (2012).

[59] H. Lesnard, M.-L. Bocquet, and N. Lorente, J. Am. Chem. Soc. 129, 4298 (2007).

[60] J. N. Ladenthin, T. Frederiksen, M. Persson, J. C. Sharp, S. Gawinkowski, J. Waluk, and T. Kumagai, Nat. Chem. 8, 935 (2016).

[61] N. Bartels, K. Golibrzuch, C. Bartels, L. Chen, D. J. Auerbach, A. M. Wodtke, and T. Schäfer, Proc. Natl. Acad. Sci. U.S.A. 110, 17738 (2013).

[62] K. H. Kramer and R. B. Bernstein, J. Chem. Phys. 42, 767 (1965)

[63] http://www.archer.ac.uk 